\title{
Thromboprophylaxis in Abdominoplasty: Efficacy and Safety of a Complete Perioperative Protocol
}

\author{
Giovanni Francesco Marangi, Francesco Segreto, Igor Poccia, Stefano Campa, Daniele Tosi, \\ Daniela Lamberti, Paolo Persichetti \\ Department of Plastic, Reconstructive and Aesthetic Surgery, Campus Bio-Medico di Roma University, Rome, Italy
}

Background Venous thromboembolism, a spectrum of diseases ranging from deep venous thrombosis to pulmonary embolism, is a major source of morbidity and mortality. The majority of cases described in plastic surgery involve abdominoplasty. Risk assessment and prophylaxis are paramount in such patients. General recommendations were recently developed, but the evidence in the literature was insufficient to prepare exhaustive guidelines regarding the medication, dosage, timing, or length of the prophylaxis.

Methods A thromboprophylaxis protocol was developed for patients undergoing abdominoplasty. The protocol consisted of preoperative, intraoperative, and postoperative measures. Enoxaparin was administered as chemoprophylaxis in selected patients. The study involved 253 patients. The patients were analyzed for age, body mass index, enoxaparin dosage, risk factors, and complications.

Results Deep venous thrombosis was documented in two cases $(0.8 \%)$. No pulmonary embolism occurred. Three patients (1.2\%) presented mild subcutaneous abdominal hematoma within the first postoperative week that spontaneously resorbed with neither aesthetic nor functional complications. Two patients $(0.8 \%)$ presented severe hematoma requiring surgical re-intervention for drainage and hemostasis revision. Statistical analysis showed no significant correlation between enoxaparin dosage and hematoma $(\mathrm{P}=0.18)$ or deep venous thrombosis $(P=0.61)$.

Conclusions The described thromboprophylaxis protocol proved to be effective in the prevention of thrombotic events, with an acceptable risk of hemorrhagic complications. Furthermore, it provides new evidence regarding the currently debated variables of chemoprophylaxis, namely type, dosage, timing, and length.

Keywords Abdominoplasty / Thrombosis / Venous thrombosis / Thromboembolism / Enoxaparin
Correspondence: Francesco Segreto Department of Plastic, Reconstructive and Aesthetic Surgery, Campus BioMedico di Roma University, Via Alvaro del Portillo, 200-00128 Rome, Italy Tel: +39-39-2504-0493

Fax: +39-062-2541-1936

E-mail: francescosegreto@gmail.com

No potential conflict of interest relevant to this article was reported.

Received: 18 Oct $2015 \bullet$ Revised: 24 Apr 2016 • Accepted: 24 May 2016

pISSN: 2234-6163 • elSSN: 2234-6171 • http://dx.doi.org/10.5999/aps.2016.43.4.360 • Arch Plast Surg 2016;43:360-364

\section{INTRODUCTION}

Abdominoplasty is the sixth most commonly performed procedure in cosmetic surgery, with 117,158 cases in 2014 in the US alone [1]. Possible postoperative complications include infection, hemorrhage, neurologic and thrombotic disorders. Venous thromboembolism (VTE), a spectrum of diseases ranging from deep venous thrombosis (DVT) to pulmonary embolism (PE), 
Table 1. Venous thromboembolism prophylaxis protocol

\begin{tabular}{|c|c|c|}
\hline Preoperatively & Intraoperatively & Postoperatively \\
\hline $\begin{array}{l}\text { 1. BMl reduction to } 30 \mathrm{~kg} / \mathrm{m}^{2} \text { or less and weight } \\
\text { stability for } 6 \text { months } \\
\text { 2. Stop or reduce smoking at least } 3 \text { months before } \\
\text { surgery } \\
\text { 3. Anamnestic and clinical assessment of risk factors }\end{array}$ & $\begin{array}{l}\text { 1. Foot-to-thigh compression garments placed } \\
\text { before the induction of general anesthesia } \\
\text { 2. Knees flexed } 5 \text { to } 10 \text { degrees }\end{array}$ & $\begin{array}{l}\text { 1. Enoxaparin started } 8-12 \text { hours postoperatively, then once } \\
\text { daily for } 6 \text { days: } \\
-4,000 \mathrm{IU} \text { if BMI } \leq 25 \mathrm{~kg} / \mathrm{m}^{2} \text { and } 1 \text { or more risk factors } \\
\text { or } 26 \mathrm{~kg} / \mathrm{m}^{2} \leq \mathrm{BMI} \leq 30 \mathrm{~kg} / \mathrm{m}^{2} \text { and weight }<75 \mathrm{~kg} \\
-6,000 \mathrm{IU} \text { if: } 26 \mathrm{~kg} / \mathrm{m}^{2} \leq \mathrm{BMl} \leq 35 \mathrm{~kg} / \mathrm{m}^{2} \\
-8,000 \mathrm{IU} \text { if } \mathrm{BMI}>35 \mathrm{~kg} / \mathrm{m}^{2} \\
\text { 2. Slightly flexed knees and repeated flexing of the foot three } \\
\text { times per hour during postoperative day } 1 \\
\text { 3. Mobilization of the patient and removal of the compressive } \\
\text { garments on postoperative day } 2\end{array}$ \\
\hline
\end{tabular}

is a major source of morbidity and mortality for these hospitalized patients. The majority of DVT cases described in the plastic surgery literature involve abdominoplasty [2]. As a consequence, DVT risk assessment and adequate prophylaxis are of paramount importance. In 2012, the ASPS Venous Thromboembolism Task Force Report developed recommendations for thromboembolism prevention [3] in the plastic surgery setting. Nevertheless, as stated by Murphy et al. [3], there is currently a lack of evidence regarding the type, dosage, timing, and length of the prophylaxis. The aim of the present study is to address these variables by describing our protocol and its outcomes in 253 abdominoplasty patients.

\section{METHODS}

The comprehensive DVT prophylaxis protocol was applied to 253 patients undergoing abdominoplasty (conventional abdominoplasty with rectus muscle plication [4]) at the Department of Plastic Reconstructive and Aesthetic Surgery of our institution between January 2008 and January 2013 (Table 1). Patients with a body mass index (BMI) between 30 and 40 were not considered eligible for surgery until the BMI was reduced to 30 or less, except in cases of severe abdominal hernia or with high risk of strangulation. Preoperatively, the patient underwent a complete physical examination with special attention to the presence of varicose veins or swollen legs; furthermore, anamnesis focused on previous abortions, inherited thrombophilic and metabolic diseases (if known), as well as on drug intake. Intraoperatively, all patients underwent passive mechanical thromboprophylaxis, and the knees were flexed to facilitate blood flow through the popliteal veins. Postoperative measures and criteria for enoxaparin administration are described in Table 1. Patients clinically suspected of developing DVT underwent ultrasound color Doppler sonography. Clinical suspicion was defined by the onset of swelling, pain, warmth, or redness in one leg. Pa-
Table 2. Enoxaparin dosage

\begin{tabular}{|lc|}
\hline No. of patients & Enoxaparin dosage (IU) \\
\hline 106 & 0 \\
92 & 4,000 \\
51 & 6,000 \\
4 & 8,000 \\
\hline
\end{tabular}

tients were analyzed for age, BMI, enoxaparin dose, risk factors (oral contraceptive or estrogen therapy intake, hypertension, smoking, diabetes) and complications (hematoma, thrombosis, seroma, wound dehiscence). Follow-up ranged from 6 months to 5 years. Statistical analyses were performed with IBM SPSS ver. 20 (IBM Corp., Armonk, NY, USA). Spearman's correlation coefficient was performed to test the possible correlations among age, BMI, enoxaparin administration, risk factors, and complications; Fisher's exact test was performed to test the possible correlations between complications and risk factors or enoxaparin administration; the null hypothesis of the Mann-Whitney U test was that the distribution of complications was the same among the enoxaparin dosages and vice versa; univariate analysis of variance was performed with the complications groups. Only Pvalues lower than 0.05 were considered as statistically significant.

\section{RESULTS}

The protocol was applied to 253 abdominoplasty patients. The mean age was 44 (ranging from 21 to 72), the average BMI was 26.4 (ranging from 17 to 40 ). Among the patients, 166 (65.6\%) had previously undergone a bariatric procedure. Nineteen patients $(7.5 \%)$ had a previous diagnosis of diabetes, 19 (7.5\%) suffered from hypertension, 77 (30.4\%) smoked, 44 (17.3\%) took hormonal therapy. Mean operative time was 115 minutes. Regarding drug administration, 144 patients received enoxaparin, while 106 did not (Table 2). Complications are reported in 


\section{Table 3. Complications}

\begin{tabular}{|lc|}
\hline Complication & $\begin{array}{c}\text { No. of patients } \\
(\%)\end{array}$ \\
\hline Deep venous thrombosis & $2(0.8)$ \\
Pulmonary embolism & 0 \\
Uncomplicated mild subcutaneous hematoma needing no & $3(1.2)$ \\
$\quad$ further treatment & $2(0.8)$ \\
Severe hematoma requiring surgical re-intervention & $1(0.4)$ \\
Seroma & $2(0.8)^{\text {a) }}$ \\
Skin necrosis & \\
\hline a) $2 \times 2$ and $2 \times 5 \mathrm{~cm}$. & \\
\hline
\end{tabular}

Table 3. DVT was documented by the mean of ultrasound color Doppler sonography in two $(0.8 \%)$ of the four patients who were clinically suspected (both gastrocnemius veins of the left leg were involved in one patient, while the lateral gastrocnemius vein of the left leg and ipsilateral sapheno-femoral junction were involved in the other one). Statistical analysis showed no significant correlation between enoxaparin administration and hematoma $(P=0.07)$, DVT $(P>0.99)$, seroma $(P>0.99)$, or necrosis $(\mathrm{P}=0.17)$. Consistently, there was no correlation between enoxaparin dosage and hematoma $(\mathrm{P}=0.18)$, DVT $(\mathrm{P}=0.61)$, seroma $(P=0.7)$, or necrosis $(P=0.12)$. No statistically significant correlation was found between any risk factor and complications.

\section{DISCUSSION}

The number of patients suffering from excess skin after massive weight loss is dramatically increasing in western societies, causing physical, psychological, and social difficulties [5]. Surgical resection by abdominoplasty is the only effective treatment to reduce abdominal skin excess. As a consequence, abdominoplasty is a commonly sought after procedure in plastic surgery [1]. Several articles discussed the complications following abdominoplasty $[6,7]$ : VTE, a spectrum of diseases ranging from DVT to PE, is a major source of morbidity and mortality for these patients. DVT is a dreadful complication. It may be initially asymptomatic and then evolve into PE, with 10 percent fatal within 1 hour of first symptoms. It can also induce permanent damage to venous valves, resulting in a chronically swollen infection-prone extremity, known as post-thrombotic syndrome [8]. The majority of VTE cases described in the plastic surgery literature involve abdominoplasty [2]. In 2012, a systematic review of outcomes found VTE to be its major complication, with $2 \%-8 \%$ incidence in three retrospective studies [9]. Recently, Winocour et al. [10] reported a $0.8 \%$ incidence of suspected or confirmed VTE following a tummy tuck. While many other surgical specialties have published specific guidelines on throm- boprophylaxis, plastic surgery has not. In 2011, the American Society of Plastic and Reconstructive Surgeons proposed a task force on VTE: Murphy et al. [3] defined up-to-date recommendations but, as they stated in their article, there was not enough evidence in the literature to write exhaustive guidelines regarding the medication, dosage, timing, or length of the prophylaxis. Our thromboembolism prevention protocol specifically addresses these variables. Enoxaparin is a pharmacologically wellknown and easy-to-manage anticoagulant. Specifically, it has been shown to reduce the incidence of postoperative DVT and PE in plastic surgery patients, with contrasting results for the risk of postoperative bleeding $[11,12]$. Several other medications, such as rivaroxaban, have not been found to be safe or reliable for thromboprophylaxis in our surgical specialty [13] and, consequently, enoxaparin is the most widely used anticoagulant [3]. In our protocol, its dosage is adapted on the BMI, as this is among the strongest risk factors in this surgical setting: large epidemiologic studies have demonstrated a significant correlation between BMI and VTE risk [14]. Optimal timing of chemoprophylaxis remains controversial. As reported by Seruya and Baker [8], 50\% of deep venous thromboses start intraoperatively as a consequence of the vasodilatory effect of general anesthesia, immobilization, supine position, and personal risk factors. Colwell et al. [15] found a significant increase in thrombin generation intraoperatively. Thus, prophylaxis must be working at that time. Nevertheless, it was demonstrated that preoperative administration of chemoprophylaxis resulted in increased bleeding without improved efficacy compared to 6-hour postoperative first administration [16]. As a consequence, we used passive mechanical thromboprophylaxis intraoperatively and started enoxaparin in the early postoperative period. Furthermore, rectus muscle plication increases intra-abdominal pressure [17], thus decreasing venous return and predisposing the patient to DVT [18]; hence, enoxaparin administration cannot be limited to the early postoperative period and, in our protocol, it is prescribed for 7 days. Risk assessment is also crucial. Appropriate assessment models have been identified in several surgical specialties to guide perioperative thromboprophylaxis: the ASPS Venous Thromboembolism Task Force Report [3] selected the 2005 Caprini Risk-Assessment Module (RAM) as the reference point to stratify plastic surgery patients. This risk score was not used in our cohort of patients, as we introduced it in our clinical practice from January 2013. Nevertheless, by retrospectively analyzing data for the patients that received enoxaparin in this study, we found that all of them had a Caprini RAM score of 3 or more, which is, according to Murphy et al. [3] the cut-off to consider the use of chemoprophylaxis. We would like to highlight the concept that an adequate thromboprophylaxis protocol should 
not focus on the perioperative period only: it is paramount that the patient undergo surgery after all the modifiable risk factors have been addressed. Several studies in the literature have reported preoperative weight stability to be associated with reduced postoperative complications as well as improved aesthetic and functional outcomes [19]. Smoking cessation three months to four weeks before surgery reduces the incidence of intraoperative and postoperative cardiovascular events [20]. Moreover, in our experience, active participation of the patient in reducing his own risk factors stimulates the patient's motivational drive, thus leading to healthier lifestyle and improved postoperative weight control [21]. In 2012, a systematic review performed by Staalesen et al. [9] found that VTE following abdominoplasty had an incidence of $2 \%-8 \%$ in three studies. Hatef et al. [12] reported a $4.65 \%$ incidence of VTE events when abdominoplasty was performed as a single procedure. The protocol described here resulted in a $0.8 \%$ incidence of DVT and no PE, thus proving it effective in reducing the incidence of VTE events. VTE is a devastating complication, with high morbidity and mortality. Adequate prophylaxis is of paramount importance for patients' safety. Unfortunately, to date, there is not enough evidence in the plastic surgery literature to make all-inclusive recommendations. Specifically, the type, dosage, timing, and length of the chemoprophylaxis are currently under debate. Our protocol specifically addressed these variables. It proved to be effective in thrombotic event prevention with an acceptable risk of hemorrhagic complications. However, larger multi-center, randomized studies would be needed to elaborate definitive guidelines.

\section{REFERENCES}

1. American Society of Plastic Surgeons. Procedural statistics [Internet]. Arlington Heights, IL: American Society of Plastic Surgeons; 2015 [cited 2016 Jan 17]. Available from: http: //www.plasticsurgery.org/Documents/news-resources/ statistics/2014-statistics/plastic-surgery-statsitics-full-report.pdf.

2. Miszkiewicz K, Perreault I, Landes G, et al. Venous thromboembolism in plastic surgery: incidence, current practice and recommendations. J Plast Reconstr Aesthet Surg 2009; 62:580-8.

3. Murphy RX Jr, Alderman A, Gutowski K, et al. Evidencebased practices for thromboembolism prevention: summary of the ASPS Venous Thromboembolism Task Force Report. Plast Reconstr Surg 2012;130:168e-175e.

4. Pitanguy I, Mayer B, Labrakis G. Abdominoplasty: personal surgical guidelines. Zentralbl Chir 1988;113:765-71.

5. Biorserud C, Olbers T, Fagevik Olsen M. Patients' experi- ence of surplus skin after laparoscopic gastric bypass. Obes Surg 2011;21:273-7.

6. Iverson RE, Gomez JL. Deep venous thrombosis: prevention and management. Clin Plast Surg 2013;40:389-98.

7. Young VL, Watson ME. The need for venous thromboembolism (VTE) prophylaxis in plastic surgery. Aesthet Surg J 2006;26:157-75.

8. Seruya M, Baker SB. MOC-PS(SM) CME article: venous thromboembolism prophylaxis in plastic surgery patients. Plast Reconstr Surg 2008;122:1-9.

9. Staalesen T, Elander A, Strandell A, et al. A systematic review of outcomes of abdominoplasty. J Plast Surg Hand Surg 2012; 46:139-44.

10. Winocour J, Gupta V, Ramirez JR, et al. Abdominoplasty: Risk Factors, Complication Rates, and Safety of Combined Procedures. Plast Reconstr Surg 2015;136:597e-606e.

11. Pannucci CJ, Bailey SH, Dreszer G, et al. Validation of the Caprini risk assessment model in plastic and reconstructive surgery patients. J Am Coll Surg 2011;212:105-12.

12. Hatef DA, Kenkel JM, Nguyen MQ, et al. Thromboembolic risk assessment and the efficacy of enoxaparin prophylaxis in excisional body contouring surgery. Plast Reconstr Surg 2008;122:269-79.

13. Dini GM, Ferreira MC, Albuquerque LG, et al. How safe is thromboprophylaxis in abdominoplasty? Plast Reconstr Surg 2012;130:851e-857e.

14. Glynn RJ, Rosner B. Comparison of risk factors for the competing risks of coronary heart disease, stroke, and venous thromboembolism. Am J Epidemiol 2005;162:975-82.

15. Colwell AS, Reish RG, Kuter DJ, et al. Abdominal contouring procedures increase activity of the coagulation cascade. Ann Plast Surg 2012;69:129-33.

16. Raskob GE, Hirsh J. Controversies in timing of the first dose of anticoagulant prophylaxis against venous thromboembolism after major orthopedic surgery. Chest 2003;124:379S$385 S$.

17. Graca Neto L, Araujo LR, Rudy MR, et al. Intraabdominal pressure in abdominoplasty patients. Aesthetic Plast Surg 2006;30:655-8.

18. Ivatury RR, Diebel L, Porter JM, et al. Intra-abdominal hypertension and the abdominal compartment syndrome. Surg Clin North Am 1997;77:783-800.

19. van der BeekES, van der Molen AM, van Ramshorst B. Complications after body contouring surgery in post-bariatric patients: the importance of a stable weight close to normal. Obes Facts 2011;4:61-6.

20. Warner MA, Offord KP, Warner ME, et al. Role of preoperative cessation of smoking and other factors in postoperative 
pulmonary complications: a blinded prospective study of coronary artery bypass patients. Mayo Clin Proc 1989;64: 609-16.
21. Segreto F, Simone P, Piombino L, Persichetti P. Plastic surgery improves long-term weight control after bariatric surgery. Plast Reconstr Surg 2014;133:599e-600e. 